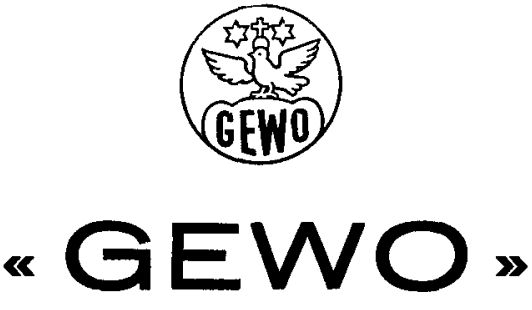

LA MARQUE DE CONFIANCE POUR SPECIALITES PHARMACEUTIQUES PRÉPAREES SUR LA BASE DE NOS PROPRES RECHERCHES

LES FILS D'ED. GEISTLICH S. A. POUR L'INDUSTRIE CHIMIQUE SECT. PHARMACEUTIQUE WOLHUSEN / SUISSE

\title{
LouIs DUPONT
}

Entreprises sanitaires

Ferblanterie et Couverture

G E N È V E

Tél. 243283

\section{GEVAERT}

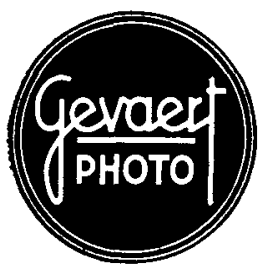

\section{CURIX - OSRAY - SCOPIX}

Le radiofilm de réputation mondiale

Photo-Produits Gevaert S. A. Belgique Représentation suisse : Gevaert Photo, Société anonyme, Bâle 6 


\section{HUGUENIN}

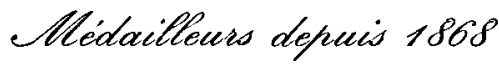

LE LOCLE SUIsoo

Insignes, Mlédailles, LPlaquettes

en excécution artistique

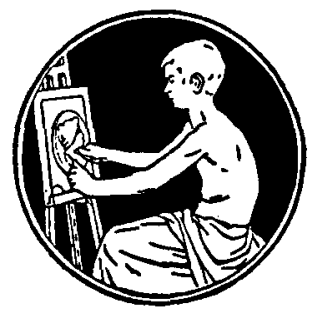

SOCIÉTÉ ANONYME

FIDUGIAIRE SUISSE

BALE Freiestrasse 90

Zurich Talstrasse 80

Genève Rue du Mont-Blanc 3

Lausanne Place St-François $14 \mathrm{~B}$

ORGANISATIONS •REVISIONS - EXPERTISES

QUESTIONS FISCALES

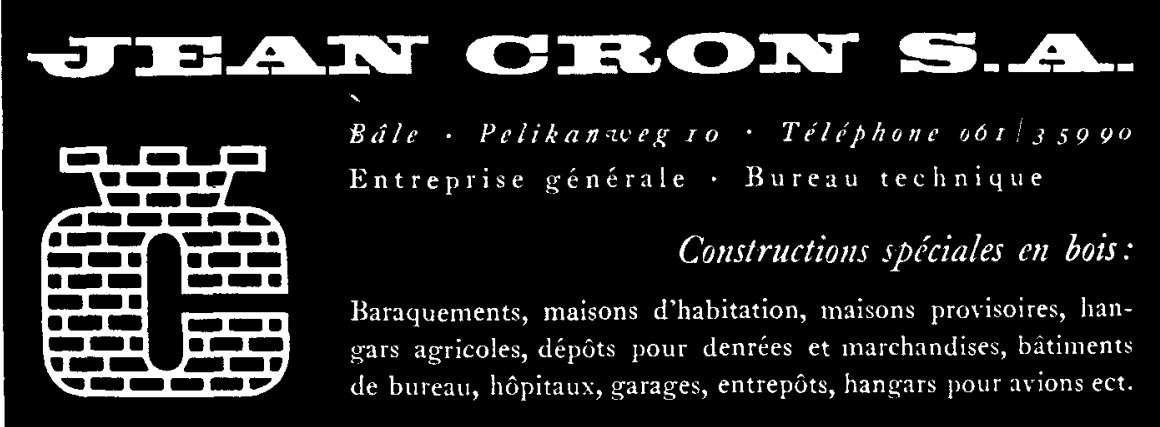



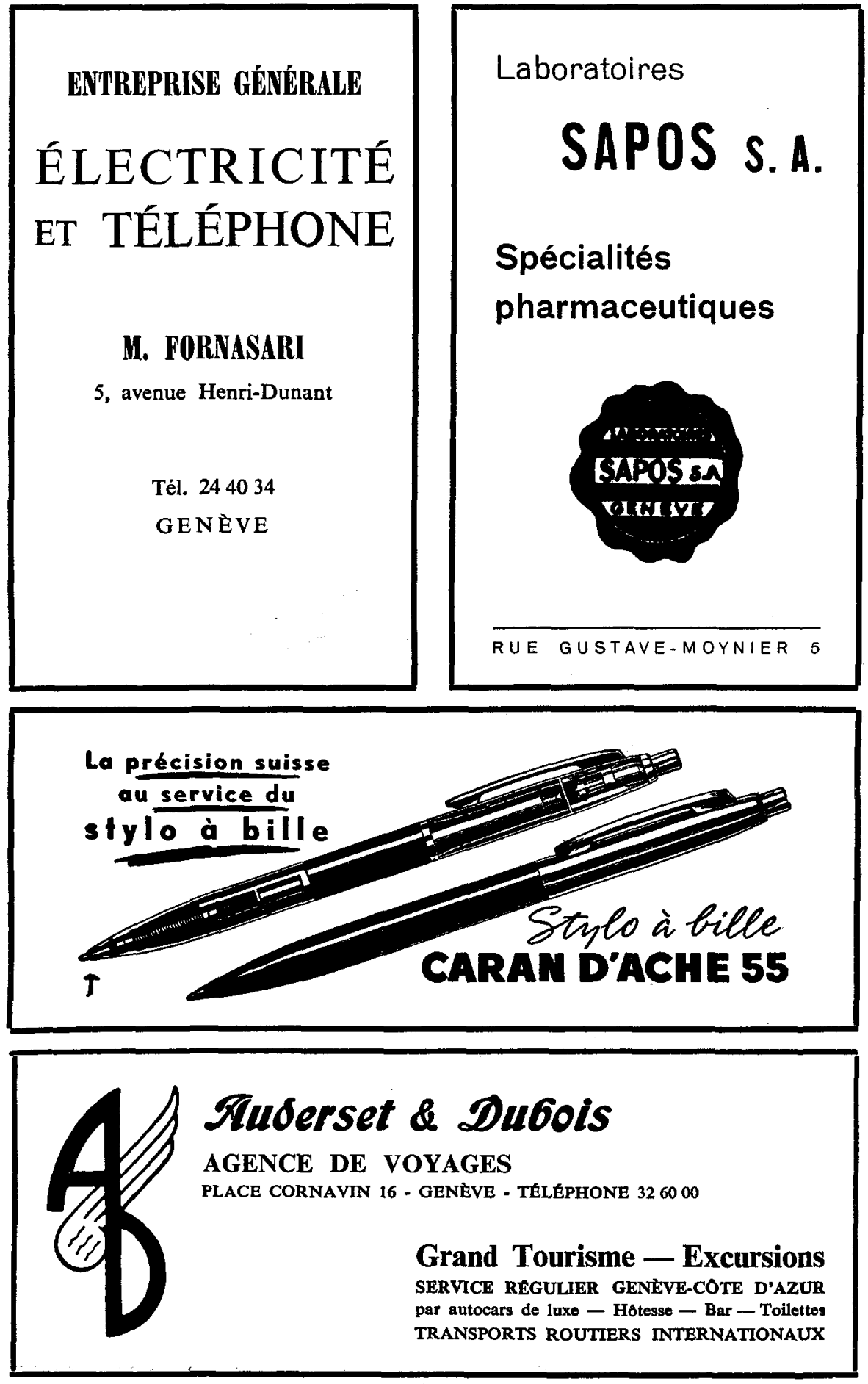


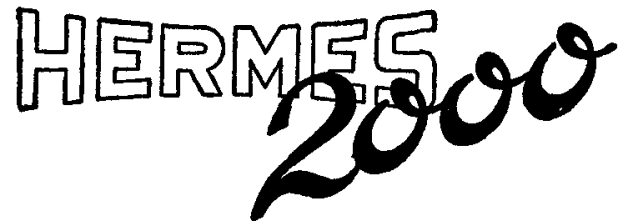

\section{LA PLUS COMPLÈTE DES MACHINES À ÉCRIRE PORTATIVES}

Margeurs éclairs

Tabulateur automatique

Réglage du toucher, eta.

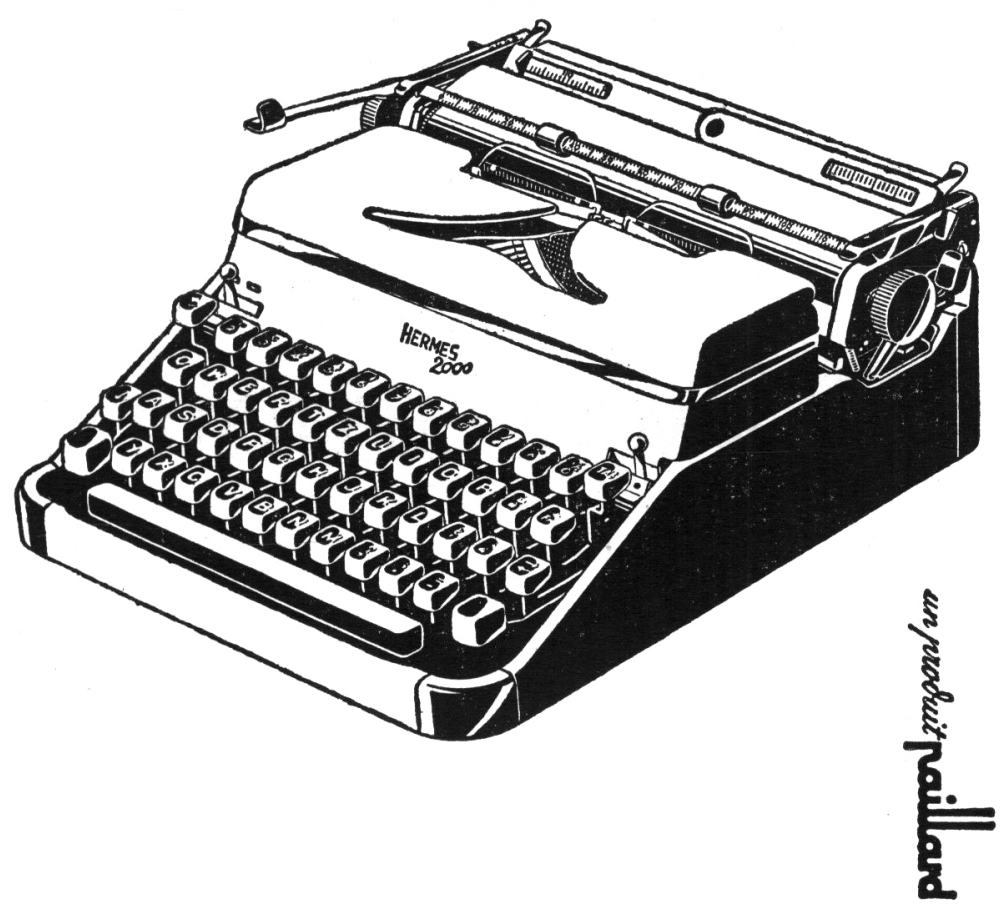

PAILLARD S.A. YVERDON/SUISSE (Fondée en 1814) 


\section{S.A. anct B. SIEGFRIED ZOFINGUE (SUISSE) • FONDÉE EN 1873}

Fabrication, depuis plus de quatre-vingts ans, de produits chimiques et pharmaceutiques d'une qualité irréprochable à l'intention des médecins et des pharmaciens

Exportation dans tous les pays de médicaments synthétiques préparés conformément aux prescriptions des diverses pharmacopées

Dérivés chimiques purs à disposition de l'industrie et pour les recherches scientifiques

Spécialités pharmaceutiques éprouvées, préparées selon les données les plus récentes de la médecine

\section{Les adresses de nos représentants à l'étranger sont fournies sur demande}


Pour Tous Vos Voyages WAGONS-LITS//COOK

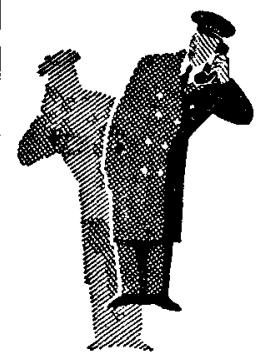

\section{Spécialistes}

du Voyage à forfait

Demandez nos brochures
A GENCES

à votre Service

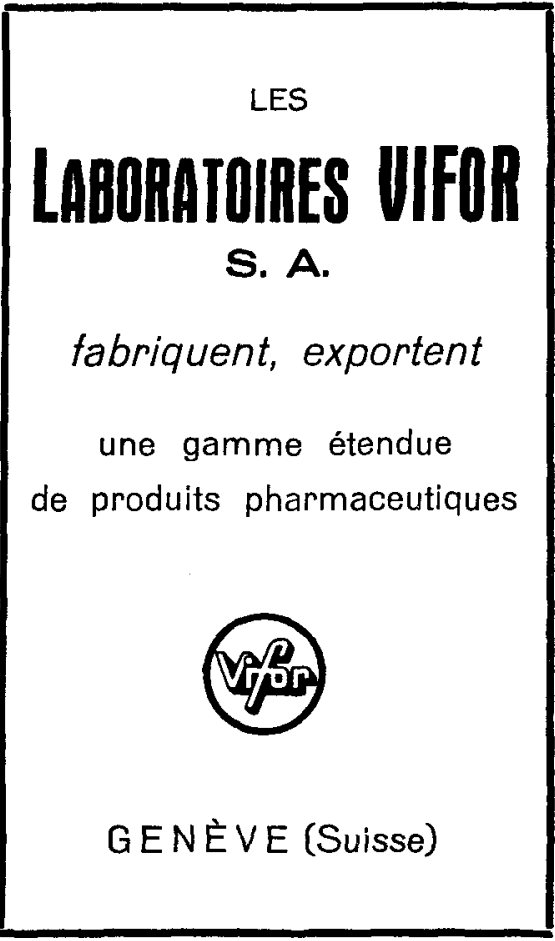

SOCIÉTÉ FIDUCIAIRE ROMANDE OFOR S.A.

- Revisions et organisations comptables

- Administration et liquidation de sociétés

- IMPOTS : Conseils par spécialiste

9, RUE D'ITALIE - GENEVE TÉLEPHONE 2432 91/3

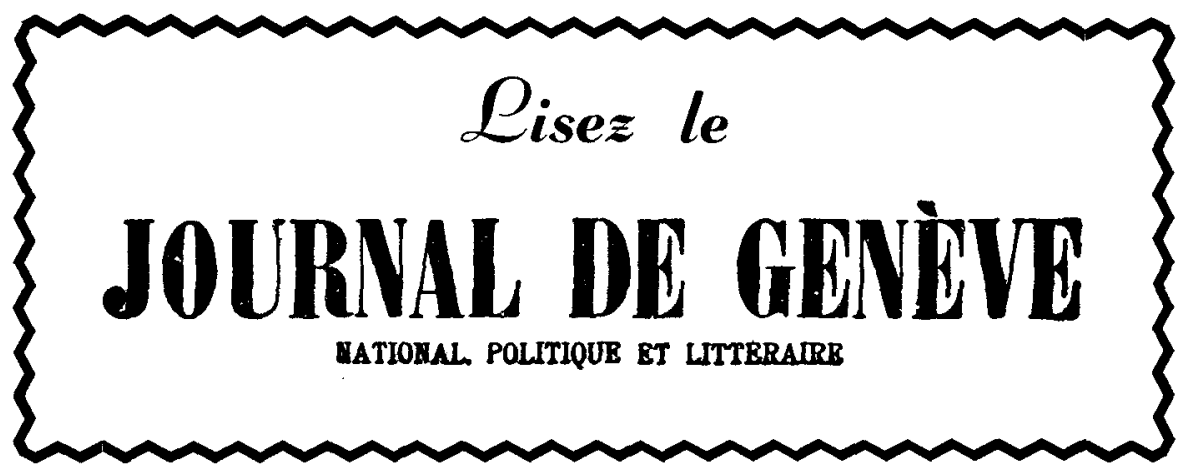




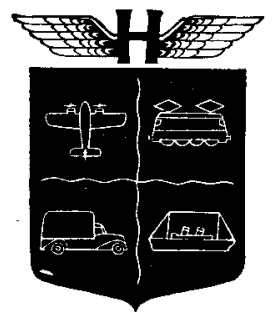

Compagnie d'Assurances Générales

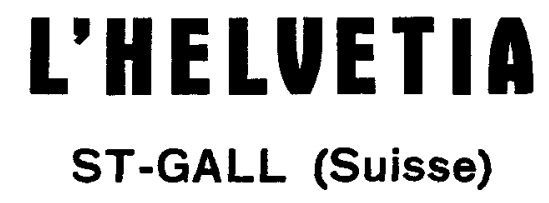

Fondée en 1858

$\begin{array}{lc} & \text { Francs suisses } \\ \text { Capital social ( } 1 / 2 \text { versé) } & 10.000 .000 \\ \text { Total des moyens de garantie } & 61.900 .000\end{array}$

\section{ASSURAMIGES TRANSPORTS}

REESSURAMICES dans toutes les Branches

Direction: ST-GALL (Suisse) Téléphone: (071) 233121

Agences en Suisse et dans beaucoup d'autres pays 


\section{BONNES ADRESSES EN SUISSE}

\section{LA RÉSIDENCE}

Florissant 11

GENEेVE

Hôtel - Restaurant - Bar

Grands et petits salons pour rtceptions 200 lits

60 salles de bains

Teléphone dans toutes les chambres

Deux tennis - Parc pour autos

Arrangements pour familles

Tel. 241380 (8 lignes)

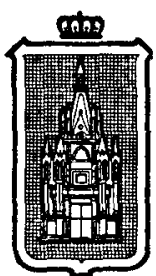

\section{HÔTEL}

RICHEIOND

GENÈVE

Nouvelle aile de luxe

Salles de réunions

Grill-Bar Le Gentilhomme

Apéritif et dîner dansants

Tél. 327120

Famille Armleder depuis 1875

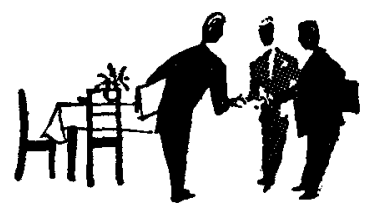

Pour leurs conférences, ils se réunissent toujours au

Buffet de la bare a ferne

F, E. KRÄHENBÜHL

Téléphone 23421
HŌ̇el du Ahône

ภी० GENEVE

Le plus récent, construit en 1950 , en plein centre. Situation tranquille au bord du Rhône, quai Turrettini. 200 chambres, toutes avec salle de bain, douches ou cabinet de toilette et radio.

Garage dans la maison Agence de vuyages.

Prix à partir de fr. 16,一, chambre, petit-déjeuner et service compris.

Tól. 327040 Tólogr.: RHOHOTEL
Préparation à

I'Universitẽ et l'Ecole Polytechnique
Section commerciale

Cours pour aides-médecins 


\section{EXTRAIT DES STATUTS}

\section{DU COMITE INTERNATIONAL DE LA CROIX-ROUGE (adoptés le 25 septembre I952)}

Article premier. - Le Comité international de la Croix-Rouge ( $C I C R$ ), fondé à Genève en I 863, consacré par les Conventions de Genève et par les Conférences internationales de la Croix-Rouge, est une institution indépendante ayant son statut propre.

Il est partie constitutive de la Croix-Rouge internationale ${ }^{\mathbf{1}}$.

ArT, 2. - En tant qu'association régie par les articles 60 et suivants du Code civil suisse, le CICR possède la personnalité civile.

ArT. 3. - Le CICR a son siège à Genève.

Il a pour emblème la croix rouge sur fond blanc. Sa devise est " Inter arma caritas".

ART. 4. - Le CICR a, notamment, pour rôle:

a) de maintenir les principes fondamentaux et permanents de la CroixRouge, à savoir : l'impartialité, une action indépendante de toute considération raciale, politique, confessionnelle ou économique, l'universalité de la Croix-Rouge et l'égalité des Sociétés nationales de la Croix-Rouge;

b) de reconnaître toute Société nationale de la Croix-Rouge nouvellement créé ou reconstituée et répondant aux conditions de reconnaissance en vigueur, et de notifier cette reconnaissance aux autres Sociétés nationales;

c) d'assumer les tâches qui lui sont reconnues par les Conventions de Genève, de travailler à l'application fidèle de ces Conventions et de recevoir toute plainte au sujet de violations alléguées des Conventions humanitaires:

d) d'agir, en sa qualité d'institution neutre, spécialement en cas de guerre, de guerre civile ou de troubles intérieurs; de s'employer en tout temps à ce que les victimes militaires et civiles desdits conflits et de leurs suites directes reçoivent protection et assistance, et de servir, sur le plan humanitaire, d'intermédiaire entre les parties;

e) de contribuer, en vue desdits conflits, à la préparation et au développement $\mathrm{du}$ personnel et du matériel sanitaires, en collaboration avec les organisations de la Croix-Rouge et les Services de santé militaires et autres autorités compétentes;

f) de travailler au perfectionnement du droit international humanitaire, à la compréhension et la diffusion des Conventions de Genève et d'en préparer les développements éventuels;

g) d'assumer les mandats qui lui sont confiés par les Conférences internationales de la Croix-Rouge.

Le CICR peut en outre prendre toute initiative humanitaire qui entre dans son rôle d'institution spécifiquement neutre et indépendante et étudier toute question dont l'examen par une telle institution s'impose.

ART. 6 (alinéa premier). - Le CICR se recrute par cooptation parmi les citoyens suisses. Le nombre de ses membres ne peut dépasser vingtcinq.

1 La Croix-Rouge internationale comprend les Sociétés nationales de la Croix-Rouge, le Comité international de la Croix-Rouge et la Ligue des Sociétés de la Croix-Rouge. L'expression - Sociétés nationales de la Croix-Rouge couvre également les Sociétés du Croissant-Rouge et la Société du Lion et Soleil Rouges. 


\section{ADRESSES DES COMITÉS CENTRAUX}

AFGHANISTAN - Croissant-Rouge afghan, Kaboul.

ALB+NIE - Croix-Rouge albanaise, 35, Rruga e Barrikadavet, Tirana.

ALLIMAGNE (République fédérale) - Croix-Rouge allemande dans la République fédérale, 71. Friedrich-EbertAllee, Bonn.

ALLEMAGNE (République démocratique) - Croix-Rouge allemande dans la République démocratique, 11, Kaitzerstrasse, Dresde.

ARGENTINE - - Croix-Rouge argentine, Hipólito Irigoyen 2068, Buenos Aires.

AUSTRALIE - Croix-Rouge australienne, 122-128, Flinders Street, Melbourne.

AUTRICHI - Croix-Rouge autrichienne, Milchgasse 1, Vienne I.

BFLGIQUE - Croix-Rouge de Belgique, 98, Chaussée de Vleurgat, Bruxelles.

BIRMANIE -- Croix-Rouge de Birmanie, Office of the director of public Health, Phayre Street, Rangoon.

BOLIVIE - Croix-Rouge bolivienne, Casilla num. 741, La Paz.

BRÉSIL - Croix-Rouge brésilienne, Praça da Cruz Vermelha, 10-12, kio de Janeiro.

BULGARIE - Croix-Rouge bulgare, 21, boul. Totleben, Sofia.

CANADA - Croix-Rouge canadienne, 95, Wellesley Street, Toronto, 5.

CFYLAN - Croix-Rouge de Cey]an, 106, Turret Road, Colombo VII.

CHILI - Croix-Rouge chilienne, Avenida Santa Maria, 0150 Correo, 15, Casilla 246. V., Santiago de Chile.

CHINE -.. Croix-Rouge chinoise, 22 Kanmein Hutung, Pékin E.

COLOMBIE - - Croix-Rouge colombienne, Carrera 5a, No 17 79, Apartado nacional 1110, Bogota.

COREE (République de) - Croix-Rouge de la République de Corée, 32 - 3 Ka Nam San Dong, Séoul.

COSIA-RICA - Croix-Rouge costaricienne, Calle 5a Sur, A partado 1025, San José.

CUBA - Croix-Rouge cubaine, Ignacio Agramonte, 461, La Havane.

DANEMAKK - Croix-Rouge danoise, Platanvej 22 , Copenthague $V$.

RIPUBI.IQUE DOMINICAINE - Croix-Rouge dominicaine, Calle Galvan, 24, Apartado 1288, Ciudad Trujillo.

EGYPTE - Société nationale du Croissant-Rouge égyptien Nahdet Masr, 34, Le Caire.

ÉQUATEUR - Croix-Rouge équatorienne, Avenida Colombia y Elizalde, 118, Ouito.

ESPAGNE - Croix-Rouge espagnole, Eduardo Dato, 18 Madrid.

E.TATS-UNIS - Croix-Rouge américaine. National Headquarters, 17th and D. Streets. N.W., Washington 13, D.C

ETHIOPIE - Croix-Rouge éthiopienne, Addis-Abeba.

FINLANDE - Croix-Rouge de Finlande, Tehtaankatu I A Helsinki.

FRANCE - Croix-Rouge française, 17, rue Quentin-Bauchart, Paris $(8 \mathrm{me}$ ).

GRANDE-BRETAGNE - Croix-Rouge britannique, 14, Grosvenor Crescent, Londres S.W.1.

GRECE - Croix-Rouge bellénique, rue Mackenzie-King, 1 et rue Solonos, 39, Athenes.

GUATÉMALA - Croix-Kouge du Guatémala, 4a. C.11-42, Zona No 1, Guatémala.

HAIITI - Croix-Rouge haïtienne, rue Férou, Port-au-Prince HONDURAS - Croix-Rouge du Honduras, Tegucigalpa HONGRIF - Croix-Rouge hongroise, Baross utca, 15 Budapest VIII.

INDE: -- Croix-Rouge de l'Inde, Red Cross Road, Neri Delhi, 2.
INDONÉSIE - Croix-Rouge indonésienne, Tanah Abang Barat, 66, Djakarta.

IRAK - Croissant-Rouge de l'Irak, Bagdad.

IRAN - Société du Lion et Soleil Rouges de l'Iran, Avenue Ark, Tébéran.

IRLANDE-Croix-Rouge irlandaise, 25 Westland Row, Dublin.

ISLANDE - Croix-Rouge islandaise. Adalstraeti $9 \mathrm{~B}$, Reykjavik.

ITALIE -- Croix-Rouge italienne, 12, Via Toscana, Rome.

JAPON - Croix-Rouge du Japon, 5, Shiba Park, Tokio.

JORDANIE - Croissant-Rouge jordanien, Amman.

LIBAN — Croix-Rouge libanaise, Beyrouth.

LIECHTENSTEIN - Croix-Rouge du Liechtenstein, Vaduz.

LUXEMBOURG - Croix-Rouge luxembourgeoise, Parc de la Ville, Luxembourg.

MEXIQUE - Croix-Rouge mexicaine, Sinaloa, $\mathrm{N}^{\circ} 20,5^{\circ}$ piso, Mexico $\mathrm{DF}$

MONACO - Croix-Rouge monégasque, Quai des Etats-Unis, Monaco.

NICARAGUA - Croix-Rouge du Nicaragua, Managua D.N.C.A.

NORVEGE - Croix-Rouge de Norvège, Parkveien, 33b, Oslo. NOUVELLE-ZÉLANDE - Croix-Rouge néo-zélandaise, 61, Dixon Street, Wellington C.I.

PAKISTAN - Croix-Rouge du Pakistan, Katrak Building Mansfield Road, Karachi $I I I$.

PANAMA - Croix-Rouge de Panama, Panama.

PARAGUAY - Croix-Rouge paraguayenne, Avenida España, 505, A suncion.

PAYS-BAS - Croix-Rouge néerlandaise, 27, Prinsessegracht, La Haye.

PÉROU - Croix-Rouge péruvienne, Tarapaca 881, Lima.

PHILIPPINES - Croix-Rouge philippine, 600 Isaac Peral Street, P.O. Box 280, Manille.

POLOGNE - Croix-Rouge polonaise, Mokotowska, 14, Varsovie.

PORTUGAL - Croix-Rouge portugaise, Secrétariat général, Jardim 9 de Abril, 1, Lisbonne.

ROUMANIE - Croix-Rouge de la République populaire roumaine, Strada Biserica Amzei, 29, Bucarest.

SAINT-MARIN - Croix-Roinge de Saint-Marin, Saint-Marin, SALVADOR Croix-Rouge du Salvador, 3a, Calle Poniente. 21, San Salvador.

SUĖDE - Croix-Rouge suédoise, Artillerigatan, 6, Stockholm 14.

SUISSE - Croix-Rouge suisse, Taubenstrasse, 8, Berne.

SYRIE - Croissant-Rouge syrien. Damas.

TCHÉCOSLOVAQUIE - Croix-Rouge tchécoslovaque Thunovska, 18, Prague III.

THAILANDE - Croix-Rouge thailandaise, King Chulalong korn Memorial Hospital, Bangkok.

TURQUIE - Société du Croissant-Rouge turc, Yenisehir, Ankara.

UNION SUD-AFRICAINE - Croix-Rouge sud-africaine, Barclay's Bank Building, 14 Holland Street, Johannesburg.

U.R.S.S. - Alliance des Sociétés de la Croix-Rouge et du Croissant-Rouge de l'U.R.S.S., Kouznetsky Most, 18/7, Moscou.

URUGUAY - Croix-Rouge uruguayenne, Avenida, 8 de Octubre, 2990, Montevideo.

VENEZUELA - Croix-Rouge vénézuélienne, Avenida de Andrés Bello, No 4, Caracas.

YOUGOSLAVIE - Croix-Rouge yougoslave, Simina ulica broj, 19, Belgrade. 